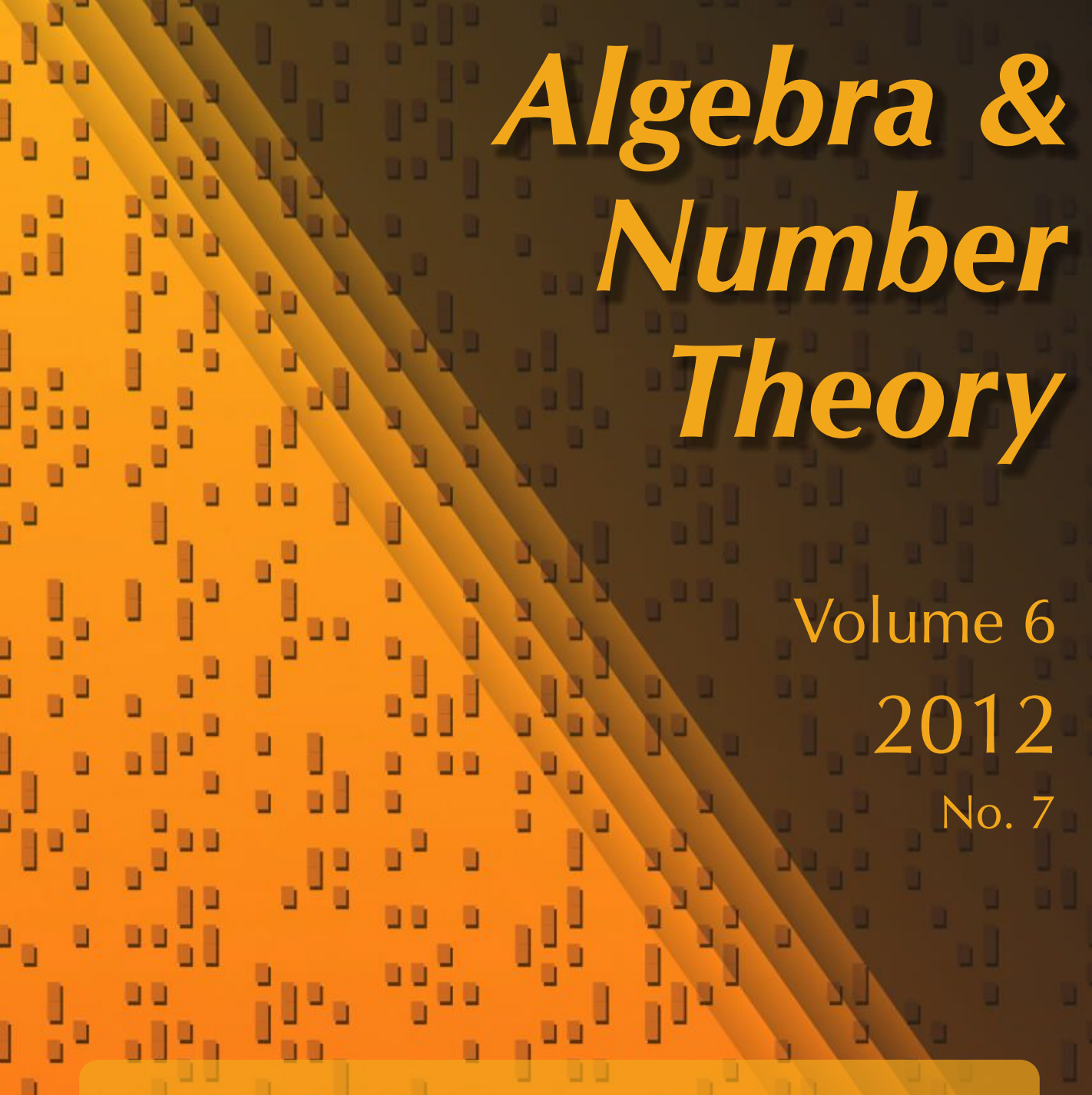




\title{
On the rank of the fibers of rational elliptic surfaces
}

Cecília Salgado

\begin{abstract}
We consider an elliptic surface $\pi: \mathscr{E} \rightarrow \mathbb{P}^{1}$ defined over a number field $k$ and study the problem of comparing the rank of the special fibers over $k$ with that of the generic fiber over $k\left(\mathbb{P}^{1}\right)$. We prove, for a large class of rational elliptic surfaces, the existence of infinitely many fibers with rank at least equal to the generic rank plus two.
\end{abstract}

\section{Introduction}

Let $k$ be a number field, $B$ a projective curve and $\pi: \mathscr{E} \rightarrow B$ an elliptic surface over $k$, that is, a projective surface endowed with a morphism $\pi$ such that almost all fibers are genus-one curves and such that there is a section $\sigma$ of $\pi$ defined over $k$ that will be fixed as the zero section. The generic fiber $\mathscr{E}_{\eta}$ is an elliptic curve over the function field $k(B)$. Since $k(B)$ is finitely generated over $\mathbb{Q}$, the Mordell-Weil Theorem is still valid in this context (Lang-Néron), and hence the set of $k(B)$-rational points of $\mathscr{E}_{\eta}$ is a finitely generated abelian group. Since all but finitely many fibers of $\pi$ are elliptic curves over the number field $k$, it is natural to be interested in comparing the rank $r$ of $\mathscr{E}_{\eta}(k(B))$ and the rank $r_{t}$ of the Mordell-Weil group of a fiber $E_{t}(k)$ for $t \in B(k)$.

A theorem on specializations by Néron [1956] or its refinement by Silverman [1994, Theorem III.11.4; 1983] in the case where the base is a curve tells us that if $\mathscr{E}$ is nonsplit, then for all but finitely many fibers we have $r_{t} \geq r$.

Billard [1998, Theorem C] showed that if we assume $\mathscr{E}$ to be $\mathbb{Q}$-rational (birational to $\mathbb{P}^{2}$ over $\mathbb{Q}$ ) and nonisotrivial, then

$$
\#\left\{t \in B(k) \mid r_{t} \geq r+1\right\}=\infty .
$$

Three natural questions arise:

1) Can we replace $\mathbb{Q}$ by an arbitrary number field $k$ and the hypothesis that $\mathscr{E}$ is $k$-rational by the hypothesis that $\mathscr{E}$ is $k$-unirational?

MSC2010: primary 14J27; secondary 11G05, 14D99.

Keywords: elliptic surface, rational surface, Mordell-Weil group, elliptic curve. 
2) Can we improve the bound, that is, have $r_{t} \geq r+2$ for infinitely many $t \in B(k)$ ?

3) Can we obtain a similar result for elliptic surfaces that are geometrically rational (that is, fixed an algebraic closure $\bar{k}$ of $k, \mathscr{E}$ is $\bar{k}$-rational) but not $k$-rational or for non(geometrically) rational elliptic surfaces, such as K3 surfaces?

We remind the reader that a geometrically irreducible algebraic variety $V$ is said to be $k$-unirational if there is a rational map of finite degree $\mathbb{P}^{n} \rightarrow V$ defined over $k$.

We will give a positive answer to question 1) and a partial answer to questions 2) and 3) in this article. Question 3) for K3 surfaces is addressed in the author's $\mathrm{Ph} . \mathrm{D}$. thesis [Salgado 2009] and will be explored in another article.

Let $X$ be a smooth projective rational surface. We denote by $\omega_{X}^{2}$ the selfintersection number of the canonical sheaf on $X$. This will be called the degree of $X$ and will be denoted by $d_{X}$ or by $d$ when the dependency on $X$ is clear. The corollary to the following theorem answers question 1).

Theorem 1.1. Let $\pi: \mathscr{E} \rightarrow B \simeq \mathbb{P}^{1}$ be a $k$-unirational elliptic surface defined over a number field $k$. There is a curve $C \rightarrow B$ such that $C \simeq_{k} \mathbb{P}^{1}$ and

$$
\operatorname{rank} \mathscr{E}_{C}(k(C)) \geq \operatorname{rank} \mathscr{E}(k(B))+1,
$$

where $\mathscr{E}_{C}=\mathscr{E} \times{ }_{B} C$.

Since the curve $C$ has infinitely many $k$-rational points, an application of Néron's or Silverman's Specialization Theorem yields the following corollary.

Corollary 1.2. Let $\pi: \mathscr{E} \rightarrow$ B be a k-unirational elliptic surface. Then

$$
\#\left\{t \in B(k) \mid r_{t} \geq r+1\right\}=\infty .
$$

Remark 1. Since rational surfaces of degree $d_{X} \geq 3$ such that $X(k) \neq \varnothing$, and del Pezzo surfaces of degree 2 having a $k$-rational point outside a certain divisor are always $k$-unirational, we conclude, from the remark above, that the class of rational elliptic surfaces to which Theorem 1.1 applies is quite large. For example, it contains all rational elliptic surfaces defined over $k$ with three distinct types of reducible fiber and/or a fiber with a double component not of type $I_{0}^{*}$. But surfaces with generic rank over $k$ equal to zero and no reducible fibers always have as $k$-minimal models del Pezzo surfaces of degree one and are therefore excluded from the hypothesis of Theorem 1.1.

Remark 2. Showing a result such as the above corollary for a rational elliptic surface having a del Pezzo surface of degree one as a $k$-minimal model is equivalent to showing that the $k$-rational points on $X$ are Zariski dense, a well known open problem. In [Ulas 2008] one can find partial results towards that direction.

The following theorems, or more precisely their corollary, answer question 2) after strengthening the hypothesis of Theorem 1.1. In order to state them we 
introduce the following terminology: if $f: X \rightarrow Y$ is a birational morphism of surfaces, passing to an algebraic closure, it is composed of monoidal transformations or blow ups of points; we will refer to the set of $\bar{k}$-points where $f^{-1}$ is not defined as the blow up locus of $f$. We will denote it by $\operatorname{Bl}(f)$. Note that if $X$ is a rational elliptic surface and $Y$ a rational model obtained after contracting $(-1)$-curves, then $\mathrm{Bl}(f)$ contains $d_{Y}$ not necessarily distinct points, thus \# $\mathrm{Bl}(f) \leq d_{Y}$ where \# denotes the number of distinct points. If $f$ is defined over $k$, the set $\mathrm{Bl}(f)$ is composed of $\operatorname{Gal}(\bar{k} \mid k)$-orbits.

Theorem 1.3. Let $\pi: \mathscr{E} \rightarrow B \simeq \mathbb{P}^{1}$ be a rational elliptic surface defined over a number field $k$ such that there is a k-birational morphism $f: \mathscr{E} \rightarrow \mathbb{P}^{2}$ in which the zero section of $\mathscr{E}$ is contracted to a point $p_{1} \in \mathbb{P}^{2}(k)$. Suppose that the blow up locus of $f$ contains at least one orbit distinct from the one given by $p_{1}$ whose points are, together with $p_{1}$, in general position. Suppose also that $\mathscr{E}$ has at most one nonreduced fiber.

Then there exists a finite covering $C \rightarrow B$ such that $C(k)$ is infinite and the surface $\mathscr{E}_{C}=\mathscr{E} \times{ }_{B} C$ satisfies

$$
\operatorname{rank} \mathscr{E}_{C}(k(C)) \geq \operatorname{rank} \mathscr{E}(k(B))+2 .
$$

Remark 3. It is simple to construct examples of rational elliptic surfaces satisfying the hypothesis of Theorem 1.3. Let $f$ and $g$ be two arbitrary cubics in $\mathbb{P}^{2}$ whose equations have coefficients in $k$. Suppose that they pass through two $k$-rational points $p_{1}$ and $p_{2}$. Then the elliptic surface given by the blow up of the intersection locus of $f$ and $g$ is certainly in this class.

Remark 4. The assumption that $\mathscr{E}$ has at most one nonreduced fiber excludes only one configuration of singular fibers, namely, $\left(I_{0}^{*}, I_{0}^{*}\right)$. This case is left out because the surface might become trivial after a quadratic base change; see Lemma 2.1.

The result above also holds for some rational elliptic surfaces whose $k$-minimal models, after contracting the zero section, are not isomorphic to $\mathbb{P}^{2}$ but to other rational surfaces defined over $k$. These are the subject of the next theorem.

Theorem 1.4. Let $\pi: \mathscr{E} \rightarrow B \simeq \mathbb{P}^{1}$ be a rational elliptic surface defined over a number field $k$. Suppose $\mathscr{E}$ does not have reducible fibers. Let $X$ be a $k$-minimal model of $\mathscr{E}$ such that there exists a birational morphism $f: \mathscr{E} \rightarrow X$ in which the zero section of $\mathscr{E}$ is contracted. Suppose that $X$ has degree $d$ and satisfies one of the following:

i) $d=4,5$ or 8 .

ii) $d=6, \# \mathrm{Bl}(f)=6$ and the largest $\mathrm{Gal}(\bar{k} \mid k)$-orbit in it has at most four points. 
Then there exists a finite covering $C \rightarrow B$ such that $C(k)$ is infinite and the surface $\mathscr{E}_{C}=\mathscr{E} \times{ }_{B} C$ satisfies

$$
\operatorname{rank}_{\mathscr{E}} \mathscr{E}_{C}(k(C)) \geq \operatorname{rank} \mathscr{E}(k(B))+2 .
$$

Elliptic surfaces satisfying the hypothesis of the previous theorem are always the blow up of a del Pezzo surface $Y$. Indeed, let $Y$ be the surface obtained after contracting the zero section of $\mathscr{E}$. Since $Y$ is a rational surface, all we have to check is that $-K_{Y}$ is ample. In fact, the anticanonical divisor of $Y$ satisfies $\left(-K_{Y}\right)^{2}>0$ and $-K_{Y} \cdot D>0$. The former because $K_{\mathscr{E}}^{2}=0$ and $Y$ is obtained by contracting a curve in $\mathscr{E}$. The latter follows from the fact that $\mathscr{E}$ has no reducible fibers and thus $-K_{\mathscr{E}} . C \geq 0$ for all $C \in \operatorname{Div}(\mathscr{E})$ with equality if and only if $C \equiv-K_{\mathscr{E}}$. By the Nakai-Moishezon Theorem $-K_{Y}$ is ample.

Once again an application of Néron-Silverman's Specialization Theorem yields the following corollary to Theorems 1.3 and 1.4.

Corollary 1.5. Let $\pi: \mathscr{E} \rightarrow B$ be an elliptic surface as in Theorem 1.3 or 1.4. For $t \in B(k)$, let $r_{t}$ be the rank of the fiber above the point $t$ and $r$ the generic rank. Then

$$
\#\left\{t \in B(k) \mid r_{t} \geq r+2\right\}=\infty .
$$

Remark 5. Since geometrically, that is, over $\bar{k}$, a rational elliptic surface is isomorphic to the blow up of nine not necessarily distinct points in $\mathbb{P}^{2}$ (see Proposition 2.2), a $k$-minimal model $X$ of a rational elliptic surface satisfies $1 \leq \omega_{X}^{2} \leq 9$. As we suppose that the elliptic surface always has a section defined over the base field $k$ which is contractible, $X$ also verifies $X(k) \neq \varnothing$.

Remark 6. Theorems 1.3 and 1.4 are valid for a larger class of rational elliptic surfaces. The choice of the cases stated was made for the sake of simplicity. The reader is invited to consult the appendix for examples of cases to which the conclusion of Theorems 1.3 and 1.4 still applies.

This text is divided as follows: Sections 2 and 3 contain geometric and arithmetic preliminaries, respectively. Section 4 is dedicated to the proof of Theorem 1.1. It contains a key proposition that reduces the proof of this theorem to the construction of a linear pencil of genus zero curves defined over the base field $k$. The proofs of Theorems 1.3 and 1.4 are given in Section 5, where we give a case-by-case construction of two pencils of curves of genus zero satisfying certain geometric conditions. The last section sheds some light from analytic number theory into the problem. There, we combine the results obtained in this article with analytic conjectures to get better, but conditional, bounds for the ranks. 


\section{Geometric preliminaries}

2A. Base change. Let $\pi: \mathscr{E} \rightarrow B$ be an elliptic surface endowed with a section and $\iota: C^{\prime} \subset \mathscr{E}$ an irreducible curve. Let $v: C \rightarrow C^{\prime}$ be its normalization. If $C^{\prime}$ is not contained in a fiber, the composition $\varphi=\pi \circ \iota \circ v$ is a finite covering $\varphi: C \rightarrow B$.

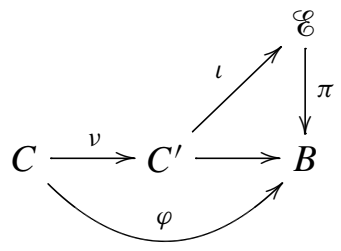

We obtain a new elliptic surface by taking the following fibered product:

$$
\pi_{C}: \mathscr{E}_{C}=\mathscr{E} \times{ }_{B} C \rightarrow C .
$$

Each section $\sigma$ of $\mathscr{E}$ naturally induces a section in $\mathscr{E}_{C}$ :

$$
(\sigma, \mathrm{id}): C=B \times{ }_{B} C \rightarrow \mathscr{E} \times{ }_{B} C .
$$

We call these sections old sections. The surface $\mathscr{E}_{C}$ has also a new section given by

$$
\sigma_{C}^{\text {new }}=(\iota \circ \nu, \mathrm{id}): C \rightarrow \mathscr{E} \times{ }_{B} C .
$$

Remark 7. Given a smooth elliptic surface $\mathscr{E}$, the elliptic surface obtained after a base change of $\mathscr{E}$ is not necessarily smooth. When this is the case, we will replace the base-changed surface by its relatively minimal model without further notice.

Remark 8. If $C$ is not contained in a fiber nor in a section, then the new section is different from the old ones, but it is not necessarily linearly independent in the Mordell-Weil group.

Remark 9. Since we want $B(k)$ to be infinite, we are naturally led to consider the base curves $B$ such that $B \simeq \mathbb{P}^{1}$, or with geometric genus $g(B)=1$ and rank $B(k) \geq 1$.

If $\mathscr{E}$ is a rational elliptic surface then, in general, after a quadratic base change we obtain an elliptic K3 surface $\mathscr{E}^{\prime}$; nevertheless, if $\mathscr{E}$ has a nonreduced fiber, that is, a fiber of type $*$, and the base change is ramified above the place corresponding to this fiber and above the place corresponding to a reduced fiber, then the base-changed surface $\mathscr{E}^{\prime}$ is still rational.

Lemma 2.1. Let $\mathscr{E} \rightarrow B$ be a rational elliptic surface. Let $\varphi: C \rightarrow B$ be a degree-two morphism where $C$ is rational. Then one of the following occurs:

i) $\mathscr{E}$ has a nonreduced fiber, that is, a fiber of type $*$, and the morphism $\varphi$ is ramified above the place corresponding to it and above the place corresponding to a reduced fiber. In this case, $\mathscr{E}_{C}=\mathscr{E} \times{ }_{B} C$ is a rational elliptic surface. 
ii) $\mathscr{E}$ has two nonreduced fibers, which are necessarily of type $I_{0}^{*}$, and the morphism $\varphi$ is ramified above both nonreduced fibers. In this case $\mathscr{E}_{C} \simeq E \times D$ where $E$ is an elliptic curve and $D$ is a curve of genus zero.

iii) $\mathscr{E}_{C}$ is a $K 3$ surface.

Proof. Let $v$ be a place of $B$ and $w$ be a place of $C$ above it. If $w$ is not ramified, then the type of the fiber $\left(\mathscr{E}_{C}\right)_{w}$ is the same as that of $(\mathscr{E})_{v}$. In this case, since the degree of $\varphi$ is two, there are two places $w, w^{\prime}$ above $v$. If $w$ ramifies above $v$ and $\mathscr{E}_{v}$ is a singular fiber, then fiber type changes, namely:

a) A fiber $(\mathscr{E})_{v}$ of type $*$ induces a fiber $\left(\mathscr{E}_{C}\right)_{w}$ whose contribution is $d_{w}=2 d_{v}-12$, where $d_{v}$ denotes the contribution of $(\mathscr{E})_{v}$, that is, the local Euler number of $\mathscr{E}_{v}$.

b) A reduced fiber, that is, a fiber $(\mathscr{E})_{v}$ of type $I_{n}, I I, I I I$ or $I V$ transforms to a fiber $\left(\mathscr{E}_{C}\right)_{w}$ whose contribution to the Euler number of the surface is $d_{w}=2 d_{v}$.

Since $\mathscr{E}$ is rational, we have

$$
\sum_{v \in B} d_{v}=12
$$

Thus if $\mathscr{E}$ has a fiber, $(\mathscr{E})_{v}$, of type $*$, and $\varphi$ is ramified above the place corresponding to it and above the place corresponding to a reduced fiber, then the Euler number of $\mathscr{E}_{C}$ is $\sum_{w \in C} d_{w}=2 d_{v}-12+2\left(12-d_{v}\right)=12$, implying that $\mathscr{E}_{C}$ is rational.

If $\mathscr{E}$ has two nonreduced fibers $\mathscr{E}_{v_{1}}$ and $\mathscr{E}_{v_{2}}$, then since each contributes at least 6 to the Euler number, which is 12, we have that the contribution of each must be exactly 6 . This implies that each nonreduced fiber is of type $I_{0}^{*}$ and moreover, that these are the only singular fibers of $\mathscr{E}$. If $\varphi$ ramifies above both $\mathscr{E}_{v_{1}}$ and $\mathscr{E}_{v_{2}}$ then by a) they both become nonsingular fibers. Since these were the unique singular fibers, the base-changed surface $\mathscr{E}_{C}$ has only smooth fibers. This entails $\mathscr{E}_{C} \simeq E \times D$ where $E$ is an elliptic curve and $d$ is a curve of genus zero.

Otherwise, that is, if $\varphi$ is ramified only above reduced fibers, then the Euler number of $\mathscr{E}_{C}$ is $2 d_{v}+2\left(12-d_{v}\right)=24$, and hence $\mathscr{E}_{C}$ is a K3 surface.

2B. Construction of rational elliptic surfaces. Let $F$ and $G$ be two distinct cubic curves in $\mathbb{P}^{2}$. We will also denote by $F$ and $G$ the two homogeneous cubic polynomials associated to these curves. Suppose $F$ is smooth.

The pencil of cubics generated by $F$ and $G$,

$$
\Gamma:=\left\{t F+u G \mid(t: u) \in \mathbb{P}^{1}\right\},
$$

has nine base points (counted with multiplicities), namely the intersection points of the curves $F$ and $G$. The blow up of these points in $\mathbb{P}^{2}$ defines a rational elliptic surface $\mathscr{E}_{\Gamma}$. 
Conversely, over an algebraically closed field, we have the following proposition. Proposition 2.2 [Miranda 1980]. Over $\bar{k}$, every rational elliptic surface with a section is isomorphic to a surface $\mathscr{E}_{\Gamma}$ for a pencil of cubics $\Gamma$ as above.

This proposition motivates the following definition.

Definition 2.3. Let $\mathscr{E}$ be a rational elliptic surface. We say that a cubic pencil $\Gamma$ in $\mathbb{P}^{2}$ induces $\mathscr{E}$ if $\mathscr{E}$ is $\bar{k}$-isomorphic to $\mathbb{P}^{2}$ blown up at the base locus of $\Gamma$.

The choice of the pencil $\Gamma$ is noncanonical.

Suppose $\Gamma$ induces $\mathscr{E}$ and $p_{1}, \ldots, p_{r}$ are the distinct base points of $\Gamma$. The Picard group of $\mathscr{E}$ is generated by the strict transform of a cubic in $\Gamma$, the exceptional curves above each $p_{i}$ and some of the (-2)-curves obtained in the process of blowing up the $p_{i}$ in case the points have multiplicity strictly larger than one as base points or are in a nongeneral position (for example, three on a line or six on a conic). As the exceptional curves are the sections of the elliptic fibration, the above gives us the following information about the (geometric) Mordell-Weil group of $\mathscr{E}$.

Lemma 2.4. Let $\Gamma$ be a pencil of cubics in $\mathbb{P}^{2}$ and $\mathscr{E}$ the elliptic surface induced by $\Gamma$. Let $s$ be the number of distinct base points of $\Gamma$ and $\bar{r}$ be the geometric MordellWeil rank of $\mathscr{E}$, that is, the rank of the Mordell-Weil group over the algebraic closure $\bar{k}$. Then $\bar{r} \leq s-1$.

Over a number field, a rational elliptic surface may have a minimal model other than $\mathbb{P}^{2}$. Hence we cannot assure the existence of a $k$-birational morphism between $\mathscr{E}$ and $\mathbb{P}^{2}$. We treat this situation in Section 3A.

2C. Néron-Tate height in elliptic surfaces. In [Shioda 1990], Shioda developed the theory of Mordell-Weil lattices. He remarked that the Néron-Severi and the Mordell-Weil groups modulo torsion have a lattice structure endowed with a pairing given essentially by the intersection pairing on the surface. In the Mordell-Weil group it coincides with the Néron-Tate height.

To define this pairing we need to introduce some notation:

Let $\Theta_{v}$ be a fiber with $m_{v}$ components denoted by $\Theta_{v, i}$. Let $\Theta_{v, 0}$ be the zero component (the one that intersects the zero section) and $A_{v}=\left(\left(\Theta_{v, i} . \Theta_{v, j}\right)\right)_{1 \leq i, j \leq m_{v}-1}$ a (negative definite) matrix. The pairing is given by the following formula:

$$
\langle P, Q\rangle=\chi+(P . O)+(Q . O)-(P . Q)-\sum_{v \in R} \operatorname{contr}_{v}(P, Q),
$$

where $\chi$ is the Euler characteristic of the surface, $R$ is the set of reducible fibers, $(P . Q)$ the intersection of the sections given by $P$ and $Q$, and $\operatorname{contr}_{v}(P, Q)$ gives the local contribution at $v$ according to the intersection of $P$ and $Q$ with the fiber $\Theta_{v}$ : if $P$ intersects $\Theta_{v, i}$ and $Q$ intersects $\Theta_{v, j}$ then $\operatorname{contr}_{v}(P, Q)=-\left(A_{v}^{-1}\right)_{i, j}$ if 
$i, j \geq 1$ and $\operatorname{contr}_{v}(P, Q)=0$ if one of the sections cuts the zero component. For a table of all possible values of $\operatorname{contr}_{v}$ according to the fiber type of $\Theta_{v}$; see [Shioda 1990].

We will only use the fact that the Néron-Tate height of a section can be computed in terms of its numerical class.

\section{Arithmetic preliminaries}

3A. Minimal models over perfect fields. The theory in this subsection was developed by Enriques, Manin [1966; 1967] and Iskoviskikh [1979]. We state the main results. For proofs, we invite the reader to look at the bibliography cited above.

Theorem 3.1. Let $X$ be a smooth minimal rational surface defined over a perfect field $k$ and let $\operatorname{Pic}(X)$ denote its Picard group over $k$. Then $X$ is isomorphic to a surface in one of the following families:

\section{A del Pezzo surface with $\operatorname{Pic}(X) \simeq \mathbb{Z}$.}

II. A conic bundle such that $\operatorname{Pic}(X) \simeq \mathbb{Z} \oplus \mathbb{Z}$.

Reciprocally, if $X$ belongs to family I then it is (automatically) minimal. If $X$ belongs to family II then it is not minimal if, and only if, $d=3,5,6$ or $d=8$ and $X$ is isomorphic to the ruled surface $\mathbb{F}_{1}$. There are no minimal surfaces with $d=7$.

Some surfaces endowed with a conic fibration are at the same time del Pezzo surfaces, namely, if $d=3,5,6$ or $d=1,2,4$ and $X$ has two distinct conic fibrations, or $d=8$ and $\bar{X}=X \times_{k} \bar{k} \simeq \mathbb{P}^{1} \times \mathbb{P}^{1}$ or $\mathbb{P}^{2}$ blown up in one point.

Definition 3.2. We say that a surface $X$ is $k$-birationally trivial or $k$-rational if there is a birational map $\mathbb{P}^{2} \rightarrow X$ defined over $k$.

Theorem 3.3. Every minimal rational surface such that $d \leq 4$ is $k$-birationally nontrivial.

Theorem 3.4. Every rational surface $X$ of degree at least five such that $X(k) \neq \varnothing$ is $k$-rational and every rational surface $X$ with $d \geq 3$ and $X(k) \neq \varnothing$ is $k$-unirational.

Remark 10. A priori most $k$-minimal surfaces with $d \geq 1$ and $X(k) \neq \varnothing$ can be a $k$-minimal model of a rational elliptic surface. The condition $X(k) \neq \varnothing$ comes from the zero section that is defined over $k$ and is contracted to a $k$-rational point. We will exclude the conic bundles such that $\bar{X}=X \times_{k} \bar{k}$ is isomorphic to $\mathbb{P}\left(\mathcal{O}_{\mathbb{P}^{1}} \oplus \mathbb{O}_{\mathbb{P}^{1}}(n)\right)$ with $n \geq 3$ since these surfaces contain a curve with self intersection $-n$ and a rational elliptic surface contains no curves with self intersection $-n$ for $n \geq 3$. 
Remark 11. If a surface $X$ of degree $d=\omega_{X}^{2}$ is a $k$-minimal model of a rational elliptic surface $\mathscr{E}$, then $\mathscr{E}$ is isomorphic to the blow up of $X$ in $d$ points which form a Galois invariant set.

We finish this subsection giving a sufficient condition for $\bar{k}$-rational elliptic surfaces to be $k$-unirational.

Lemma 3.5. Let $\mathscr{E}$ be a $\bar{k}$-rational elliptic surface defined over a perfect field $k$. If $\operatorname{rank} \operatorname{Pic}(\mathscr{E} \mid k) \geq 5$ then $\mathscr{E}$ is $k$-unirational.

Proof. Since the Picard group of $k$-minimal rational surfaces has rank one or two, any $k$-minimal model of $\mathscr{E}$ will be obtained after contracting at least three $(-1)$ curves. Hence if $X$ is a $k$-minimal model of $\mathscr{E}$ then $K_{X}^{2} \geq 3$. Moreover, $X(k) \neq \varnothing$ since the image of the zero section will provide at least one $k$-rational point. It follows then from Theorem 3.4 that $\mathrm{X}$ is $k$-unirational.

3B. Kummer theory. Let $K$ be a number field or a function field and $A$ an abelian variety. Let $P \in A(K)$ be a point of infinite order. We say that $P$ is indivisible by $n$ if for all $Q \in A(K)$ such that there exists a divisor $d$ of $n$ with $[d] Q=P$, we have $d= \pm 1$.

Remark 12. In [Hindry 1988], Hindry defines a point as being indivisible if it is indivisible by all natural numbers $m>1$. One can replace this definition by the one introduced above, that is, $P$ indivisible by $m$ in the hypothesis of [Hindry 1988, Lemme 14]. In fact, let $P$ be a point indivisible by $m$. We can write $P=[l] P_{1}$ with $P_{1}$ indivisible as in [Hindry 1988] and $(l, m)=1$. There exist $u, v \in \mathbb{N}$ such that $u l+v m=1$, which allows us to write

$$
P_{1}=[u] P+[v m] P_{1},
$$

and thus

$$
K\left(\frac{1}{m} P\right) \subseteq K\left(\frac{1}{m} P_{1}\right)=K\left(\frac{u}{m} P\right) \subseteq K\left(\frac{1}{m} P\right) .
$$

Hence $K\left(\frac{1}{m} P_{1}\right)=K\left(\frac{1}{m} P\right)$. In the rest of this subsection we state some of the results that can be found in [Hindry 1988] about the degree of the extension $K\left(\frac{1}{m} P\right)$ taking this remark into account, that is, replacing the hypothesis $P$ indivisible, by the hypothesis $P$ indivisible by $m$.

Let $n \in \mathbb{N}$ and $P$ be a point indivisible by $m$ where $m$ divides $n$. Denote by $A_{n}$ the set of $n$-torsion points in $A(\bar{K})$ and $\frac{1}{m} P$ a point $Q \in A(\bar{k})$ such that $[m] Q=P$. The Galois group $G_{n, K,(1 / m) P}$ of the extension

$$
K\left(A_{n}, \frac{1}{m} P\right) \mid K\left(A_{n}\right)
$$

can be viewed as a subgroup of $A_{m}$. Kummer theory for abelian varieties tells us that if $K$ is a number field the group $G_{n, K, \frac{1}{m} P}$ is actually almost the whole group 
$A_{m}$, that is, its image inside $A_{m}$ by an injective map is of finite index which is bounded by a constant independent of $P$ and $m$.

This theory was studied in full generality by Ribet, Bertrand, Bashmakov and others. We restrict ourselves to elliptic curves. For us $k$ is a number field and $K=k(B)$ is the function field of a projective curve $B$.

We now state a typical result of Kummer theory for elliptic curves. This is valid in a more general context; see [Ribet 1979].

Theorem 3.6. Let $E$ be an elliptic curve defined over a number field $k, n \in \mathbb{N}$ and $P \in E(k)$ a point indivisible by $m$ where $m$ divides $n$. There exists a positive constant $f_{0}=f_{0}(E, k)$ such that $\left|G_{n, k,(1 / m) P}\right| \geq f_{0} . m$.

Proof. See [Hindry 1988, Appendix 2] for when $P$ is an indivisible point.

Let $\pi: \mathscr{E} \rightarrow B$ be an elliptic surface and $P$ a point indivisible by $n$ in the generic fiber. Since Galois groups become smaller after specialization, after applying the previous theorem to a fiber $E=\pi^{-1}(t)$ defined over the number field $k$, we have

$$
\left|G_{n, k(B),(1 / m) P}\right| \geq\left|G_{n, k,(1 / m) P}\right|,
$$

which gives us a theorem as Theorem 3.6 for elliptic curves over function fields.

Theorem 3.7. Let $E$ be an elliptic curve defined over a function field $K, n \in \mathbb{N}$ and $P \in E(K)$ a point indivisible by $m$ where $m$ divides $n$. There exists a positive constant $f_{0}=f_{0}(E, K)$ such that $\left|G_{n, K,(1 / m) P}\right| \geq f_{0} . m$.

Remark 13. If the surface $\mathscr{E}$ above is not isotrivial then the endomorphism ring of the generic fiber $E$ is $\mathbb{Z}$ and the result above is stronger, namely, the group $G_{n, K,(1 / m) P}$ is almost all the set of $m$-torsion points $\mathscr{E}_{m}$, that is, its image inside the $m$-torsion subgroup under an injective map is of finite index, bounded by a constant independent of $P$ and $m$; see [Hindry 1988, Proposition 1].

We finish this subsection with a lemma about torsion points on elliptic curves that will be used in the next section during the proof of Proposition 4.2. See [Serre 1972] for a proof and more results on torsion points on elliptic curves.

Lemma 3.8. Let $E$ be an elliptic curve defined over a field $k$, which is either a number field or a function field, and $P \in E(\bar{k})[m] \backslash E(k)$ a point of order $m$. Then there exists an $\alpha>0$ and a constant $c_{E, k}$ independent of the point $P$ such that

$$
[k(P): k] \geq c_{E} \cdot m^{\alpha} .
$$

\section{Proof of Theorem 1.1}

Let $\pi: \mathscr{E} \rightarrow B$ be as in Section 2. In the first subsection we show that given a nonconstant pencil of curves in $\mathscr{E}$ that are not contained in a fiber of $\pi: \mathscr{E} \rightarrow B$, then all but finitely many curves in it yield, after base change (see Section 2A), a 
new section that is independent of the old sections. The proof of Theorem 1.1 then depends only on a construction of a family of irreducible curves defined over $k$ with infinitely many $k$-rational points, that is, $\mathbb{P}^{1}$ or genus-one curves with positive Mordell-Weil rank. This construction will be given in the second subsection.

4A. A key proposition. First we state the most useful criteria for us to determine when a new section is independent of the old ones.

Lemma 4.1. An irreducible curve $C \subset \mathscr{E}$ that is not a component of a fiber induces a new section on $\mathscr{E} \times{ }_{B} C$ independent of the old ones if and only if for every section $C_{0} \subset \mathscr{E}$ and every $n \in \mathbb{N}^{*}$, the curve $C$ is not a component of $[n]^{-1}\left(C_{0}\right)$.

Let $\mathscr{C}$ be a family of curves in a projective surface $X$. We call $\mathscr{C}$ a numerical family if all its members belong to the same numerical class in the Néron-Severi group. We prove that in a numerical family it is enough to check the conditions of the lemma for a bounded $n$ and a finite number of sections. Thus if the family is infinite, all but finitely many members induce new independent sections after base change.

Proposition 4.2. Let $\mathscr{E} \rightarrow B$ be an elliptic surface defined over a number field $k$. Let $\mathscr{C}$ be a numerical family of curves inside $\mathscr{E}$. There exist an $n_{0}(\mathscr{C}) \in \mathbb{N}$ and a finite subset $\Sigma_{0}(\mathscr{C}) \subset \operatorname{Sec}(\mathscr{E})$ such that for $C$ in the family $\mathscr{C}$, the new section induced by $C$ is linearly dependent of the old ones if, and only if, $[n] C \in \Sigma_{0}$ for some $n \leq n_{0}$.

Proof. Suppose $[n] C=C_{0}$ for some section $C_{0}$. We may assume such $n$ to be minimal, that is, there does not exist $n^{\prime}<n$ such that the curve $\left[n^{\prime}\right] C$ is a section. The proof is divided in two parts: bounding $n$ from above using Kummer theory (see Section 3B), and then, for a fixed $n$ such that $C_{0}=[n] C$, showing that the set of sections in the same numerical class is finite by Néron-Tate height theory.

1) Bounding $n$ : We define the degree of a curve in $\mathscr{E}$ by its intersection with a fiber:

$$
\operatorname{deg}(C)=(C . F) .
$$

If $C_{0}$ is a section we have $\operatorname{deg}\left(C_{0}\right)=\left(C_{0} . F\right)=1$. The degree of $C$, which will be denoted by $h$, is fixed within the family, since all curves belong to the same numerical class.

The map $[n]$ is not a morphism defined on the whole surface, but on an open set $U \subseteq \mathscr{E}$ which excludes the singular points in the fibers. Since sections do not intersect the fibers in singular points, they are contained in $U$. This allows us to write

$$
\operatorname{deg}\left([n]^{-1} C_{0}\right)=\left(\left([n]^{-1} C_{0}\right) \cdot F\right)=n^{2}\left(C_{0} \cdot F\right)=n^{2} .
$$

Thus, $\lim _{n \rightarrow \infty} \operatorname{deg}[n]^{-1}\left(C_{0}\right)=\infty$.

Denote by $K$ the field $k(B)$, by $E$ the generic fiber of $\mathscr{E}$ and by $P_{0}$ the point in $E(K)$ corresponding to the section $C_{0}$. Let $P \in E(\bar{K})$ be such that $[n] P=P_{0}$ 
where $n$ is minimal with respect to the expression above. We now show that $n$ is bounded by a constant $n_{0}$ that depends only on $E, K$ and $h$.

Note first that if $P$ is a torsion point, we know by Lemma 3.8 that its order $m$ is bounded by $c_{E} \cdot m^{\alpha} \leq[K(P): K]=h$.

Now suppose $P$ is of infinite order. Let $m$ be the smallest positive integer such that there exist $P_{1} \in E(K)$ and $T$ a torsion point satisfying $[m] P=P_{1}+T$. We claim that $P_{1}$ is indivisible by $m$. If $l$ is a divisor of $m$ such that $[l] Q=P_{1}$ with $Q \in E(K)$, then

$$
[l][m / l] P=[l] Q+T
$$

and hence there exists a torsion point $T_{1}$ such that $[\mathrm{m} / \mathrm{l}] P=Q+T_{1}$. By the minimality of $m$ with respect to the equation above, we must have $l= \pm 1$.

Let $m^{\prime}=m m_{1}$ where $m_{1}$ is the order of the torsion point $T$. Let $T^{\prime}$ be such that $[m] T^{\prime}=T$ and put $P^{\prime}=P+T^{\prime}$; then $[m] P^{\prime}=P_{1}$ and $T^{\prime} \in E_{m^{\prime}}$. Applying Theorem 3.7 we obtain

$$
\left[K\left(P^{\prime}, E_{m^{\prime}}\right): K\left(E_{m^{\prime}}\right)\right] \geq m \cdot f_{1} .
$$

Now, note that $K\left(P, E_{m^{\prime}}\right)=K\left(P^{\prime}, E_{m^{\prime}}\right)$ hence

$$
h=[K(P): K] \geq\left[K\left(P, E_{m^{\prime}}\right): K\left(E_{m^{\prime}}\right)\right]=\left[K\left(P^{\prime}, E_{m^{\prime}}\right): K\left(E_{m^{\prime}}\right)\right] \geq f_{1} . m,
$$

and thus $\mathrm{m}$ is bounded. Since $T$ is defined over $K(P)$, its order $m_{1}$ is also bounded in terms of $h$, and thus $\left[m m_{1}\right] P=m_{1} P_{1} \in E(K)$ with $n \leq m m_{1}$ bounded as stated.

2) The numerical class of a section: Fix $C_{1}, \ldots, C_{r}$ generators of the Mordell-Weil group.

For a fixed $n$, the intersection multiplicity $\left(([n] C) . C_{i}\right)$ is also fixed, say equal to $n_{i}$, and it depends only on the numerical class of $C$. The same holds for the intersection of $C$ with the zero section, say equal to $m_{0}$ and for the intersection with the fiber components $\Theta_{v}$, say equal to $l_{v, j_{v}}$. The Néron-Tate height in an elliptic surface is uniquely determined by the intersection numbers above. The set

$\Sigma_{0}=\left\{\right.$ sections $\left.C_{0} \mid\left(C_{0} \cdot C_{i}\right)=n_{i}, i=1, \ldots, r,\left(C_{0} . O\right)=m_{0},\left(C_{0} \cdot \Theta_{v, j_{v}}\right)=l_{v, j_{v}}\right\}$

is finite since it is a set of points with bounded Néron-Tate height, thus there are only finitely many possible sections $C_{0}$ such that $C \subset[n]^{-1} C_{0}$.

Corollary 4.3. Let $\mathscr{E}$ be an elliptic surface and $\mathscr{L}$ a nonconstant numerical family of curves on $\mathscr{E}$ whose members are not contained in the fibers of $\mathscr{E}$. Then for almost all member $C$ of the pencil, the new section induced by $C$ is independent of the old sections.

Proof of Theorem 1.1. Let $\psi: \mathbb{P}^{2}-\rightarrow \mathscr{E}$ be a $k$-unirational map. Let $\mathscr{L}$ be given by the set of lines in $\mathbb{P}^{2}$. Then $\mathscr{L}^{\psi}=\{\psi(L) \mid L \in \mathscr{L}\}$ is an infinite family of curves in 
$\mathscr{E}$ defined over $k$, whose general member is integral and of geometric genus zero. These curves cannot be all contained in a fiber of $\mathscr{E}$ as the family is infinite, with irreducible members, and there is only a finite number of reducible fibers for the elliptic fibration in $\mathscr{E}$.

The theorem follows from an application of Corollary 4.3 to the family $\mathscr{L}^{\psi}$.

\section{Proof of Theorems 1.3 and 1.4}

To prove Theorems 1.3 and 1.4 we need to produce two families of curves defined over $k$. These families must not only have infinitely many $k$-rational points, but also be such that the fibered product of two curves in different families is irreducible. The first subsection is devoted to the construction of such families, first done in the context of Theorem 1.3, that is, over $\mathbb{P}^{2}$, then in the settings of Theorem 1.4, that is, over other $k$-minimal surfaces. The proof of irreducibility of the generic member of the constructed families is given in the second subsection and is followed by the verification that such curves contain indeed infinitely many $k$-rational points. Finally, all is assembled in Section 5D to conclude the proofs of Theorems 1.3 and 1.4.

5A. Construction of linear pencils of rational curves. The results presented in this subsection are technical and may be skipped by the reader willing to accept the existence of two linear pencils of conics, that is, copies of $\mathbb{P}^{1}$ intersecting the fibers with multiplicity two in the surfaces satisfying the hypothesis of Theorem 1.3. Here we provide "case-by-case", depending on the configuration of blown up points, constructions of linear pencils of curves on $\mathscr{E}$ to which we apply Corollary 4.3. We construct two linear pencils of rational curves defined over $k$ in a $k$-minimal model of the rational elliptic surface $\mathscr{E} \rightarrow B$. Since the base-changed surface fibers over the fibered product of these two curves over $B$, we fabricate those curves in a way that their fibered product has genus at most one. We state below sufficient conditions for this.

Lemma 5.1. Let $C_{1}$ and $C_{2}$ be two smooth projective rational curves given with two distinct morphisms $\varphi_{i}: C_{i} \rightarrow B$ of degree 2 to a genus zero curve $B$. Then $g\left(C_{1} \times{ }_{B} C_{2}\right) \leq 1$.

Proof. It is a simple application of the Hurwitz formula.

We can proceed to the constructions. They depend on the degree of the $k$-minimal model considered, as well as on the configuration of the blown up points under the action of the absolute Galois group $\operatorname{Gal}(\bar{k} \mid k)$. We start with the simplest case, namely, when $\mathscr{E}$ has a minimal model isomorphic to $\mathbb{P}^{2}$.

a) A minimal model $k$-isomorphic to $\mathbb{P}^{2}$. Let $p_{1}, \ldots, p_{9}$ be the nine not necessarily distinct points in the blow up locus. Since the zero section is defined over $k$, at least 
one of the points above is $k$-rational, say $p_{1}$. If $C$ is a curve of degree $d$ in $\mathbb{P}^{2}$ with multiplicity $m_{i}$ through $p_{i}$ then its genus satisfies

$$
g(C) \leq \frac{(d-1)(d-2)}{2}-\sum_{i} \frac{m_{i}\left(m_{i}-1\right)}{2} .
$$

Denote by $C^{\prime}$ its strict transform under the blow up $\mathscr{E} \rightarrow \mathbb{P}^{2}$. Then the degree of the map given by the restriction of $\pi$ to $C^{\prime}$ is given by

$$
\operatorname{deg}\left(C^{\prime} \rightarrow B\right)=\left(F . C^{\prime}\right)=3 d-\sum_{i} m_{i}
$$

where $F$ is a fiber of the elliptic fibration $\pi$.

Let $\mathscr{L}_{1}$ be the pencil of lines in $\mathbb{P}^{2}$ through $p_{1}$. Let $\mathscr{L}_{1}^{\prime}$ be the pencil of curves in $\mathscr{E}$ given by the strict transforms of the curves in $\mathscr{L}_{1}$ and $C^{\prime}$ a curve in $\mathscr{L}_{1}^{\prime}$ - from now on the superscript ' will denote the pencil or curve in the elliptic surface given by the strict transform of that in the minimal model. Then

$$
\operatorname{deg}\left(C^{\prime} \rightarrow B\right)=3.1-1=2 .
$$

We now construct a second pencil of rational curves $\mathscr{L}_{2}$ such that the curves in the pencil of strict transforms induced in $\mathscr{E}$ satisfy Lemma 5.1.

Since the blow up is defined over $k$, the set formed by the other points is invariant under the action of $\operatorname{Gal}(\bar{k} \mid k)$. The construction depends on the size of the smallest orbit different from $p_{1}$ whose points are, together with $p_{1}$, in general position, that is, no three are collinear, no six lie in a conic and there is no cubic through eight of the points singular at one of them.

i) One other $k$-rational point $p_{2}$.

In this case we can construct $\mathscr{L}_{2}$ in a similar way as we did for $\mathscr{L}_{1}$ : take

$$
\mathscr{L}_{2}=\left\{l \text { a line in } \mathbb{P}^{2} \text { through } p_{2}\right\} .
$$

Any curve in $\mathscr{L}_{2}^{\prime}$ together with any curve in $\mathscr{L}_{1}^{\prime}$ satisfies Lemma 5.1.

ii) Two conjugate (under $\operatorname{Gal}(\bar{k} \mid k)$ ) points $p_{2}, p_{3}$.

Let $\Lambda$ be a pencil of cubics in $\mathbb{P}^{2}$ inducing $\mathscr{E}$ such that $p_{1}, p_{2}, p_{3}$ are base points of it. Since we suppose that points are in general position, $p_{1}, p_{2}$ and $p_{3}$ are not collinear. Let us first suppose that there are no other base points and thus that the multiplicities $\left(m_{1}, m_{2}, m_{3}\right)$ of $p_{1}, p_{2}, p_{3}$ as base points of $\Lambda$ are $(1,4,4),(3,3,3),(5,2,2)$ or $(7,1,1)$. In the first case, every cubic in $\Lambda$ shares the same tangent line, say $l_{2}$, through $p_{2}$ as well as the same tangent line, say $l_{3}$, through $p_{3}$. We consider $\mathscr{L}_{2}$, the set of conics through $p_{2}, p_{3}$ with tangents $l_{i}$ through $p_{i}$, for $i=2,3$. Let $C$ be a conic in $\mathscr{L}_{2}$. Then $\mathrm{C}$ intersects the cubics of $\Lambda$ in $p_{2}$ and $p_{3}$ with intersection multiplicity two and intersects in 
two other points. Hence, the morphism from the strict transform $\varphi_{C^{\prime}}: C^{\prime} \rightarrow B$, given by the restriction of the fibration to $C^{\prime}$, has degree two. In the remaining three cases all cubics of $\Lambda$ share a tangent line, say $l_{1}$, through $p_{1}$. We take $\mathscr{L}_{2}$ to be the set of conics through $p_{1}, p_{2}, p_{3}$ with prescribed tangent $l_{1}$ through $p_{1}$. It is a linear pencil of conics, since the space of conics in $\mathbb{P}^{2}$ has dimension 5 . A conic $C$ in $\mathscr{L}_{2}$ also intersects the cubics in $\Lambda$ at the point $p_{1}$ twice, at the points $p_{2}, p_{3}$ and at two other points. Thus if $C$ is a conic in $\mathscr{L}_{2}$ then the morphism $\varphi_{C^{\prime}}$ from $C^{\prime}$ to $B$ also has degree two. As in i), any curve in $\mathscr{L}_{2}^{\prime}$ together with any curve in $\mathscr{L}_{1}^{\prime}$ satisfies the hypothesis of Lemma 5.1.

Now suppose there are other base points. If there is another orbit with two or four points one may apply construction iv) below independently of the configuration of these extra points. If there are three, five or six other conjugate base points then apply constructions iii), v), or vi), respectively, below.

iii) Three conjugate points $p_{2}, p_{3}, p_{4}$.

The construction here is simpler. The pencil of conics $\mathscr{L}_{2}$ through $p_{1}, p_{2}$, $p_{3}$ and $p_{4}$ is such that for every curve $C \in \mathscr{L}_{2}$ the morphism $\varphi_{C}^{\prime}$ has degree two.

iv) Four conjugate points $p_{2}, p_{3}, p_{4}, p_{5}$.

As in the previous case, but now we let $\mathscr{L}_{2}$ be the pencil of conics through $p_{2}, p_{3}, p_{4}$ and $p_{5}$.

v) Five conjugate points $p_{2}, p_{3}, p_{4}, p_{5}, p_{6}$.

Take $\mathscr{L}_{2}$ to be the pencil of cubics through $p_{1}, \ldots, p_{6}$ with a singularity at $p_{1}$. The degree of the morphism $\varphi_{C}$ for $C \in \mathscr{L}_{2}$ is two $(9-5-2=2)$. So that together with a curve in $\mathscr{L}_{1}^{\prime}$ the curve $C$ satisfies the hypothesis of Lemma 5.1.

vi) Six conjugate points $p_{2}, \ldots, p_{7}$.

Consider $\mathscr{L}_{2}$ to be the pencil of quintics singular at each $p_{2}, \ldots, p_{7}$ that passes through $p_{1}$ as well. Since $\operatorname{dim}\left(H^{0}\left(\mathbb{P}^{2}, \mathcal{O}(5)\right)\right)=21$ and we have imposed 19 conditions, $\mathscr{L}_{2}$ forms at least a linear pencil. The curves in it are rational since $g \leq 6-6=0$.

The degree of $\varphi_{C}^{\prime}$ for $C \in \mathscr{L}_{2}$ is equal to $5.3-6.2-1=2$.

vii) Seven conjugate points $p_{2}, \ldots, p_{8}$.

Consider $\mathscr{L}_{2}$ the space of quartics through the seven points $p_{2}, \ldots, p_{8}$ with multiplicity at least three at $p_{1}$. That gives at most 13 conditions in a space of dimension 15. So $\mathscr{L}_{2}$ is at least a linear pencil. Its curves are rational and the degree of the induced morphism to $B$ is equal to $4.3-7-3=2$.

viii) Eight conjugate points $p_{2}, \ldots, p_{9}$.

We consider highly singular curves. Take $\mathscr{L}_{2}$ to be the set of curves of degree 17 such that:

- The eight points $p_{2}, \ldots, p_{9}$ are singular with multiplicity at least six. 
- It passes through $p_{1}$.

This gives us at most 169 conditions in a 171-dimensional space and thus at least a linear pencil. The degree of the morphism is also two and the curves have genus zero as in the previous cases.

Irreducibility of the curves constructed above: Since the points considered are in general position the curves constructed above are irreducible. This is trivially verified in cases i)-iv). In cases v)-viii) one can easily check that if the pencil is generically reducible then either its base points are in nongeneral position, that is, there are three collinear points, six lie on a conic or eight on a cubic singular at one of them, or the Galois orbits break into smaller orbits.

We now focus on the other possible minimal models over $k$. As observed after the statement of Theorem 1.4, we may suppose $X$ is a del Pezzo surface. Let us first recall some geometric and arithmetic facts about those surfaces.

Since we are dealing with surfaces whose Picard group over $k$ is small (isomorphic to $\left.\mathbb{Z} . \omega_{X}\right)$, the most natural place to look for curves defined over $k$ is $H^{0}\left(X, \omega_{X}^{-n}\right)$. We now recall the dimension of these spaces and the genus of the curves in them.

Lemma 5.2. Let $X$ be a $k$-minimal del Pezzo surface of degree $1 \leq\left(\omega_{X} . \omega_{X}\right)=d \leq 8$ defined over a number field $k$. Given points $p_{1}, \ldots, p_{j}$ in $X(\bar{k})$ and nonnegative integers $n_{1}, \ldots, n_{j}$, let $\mathscr{L}=\left\{s \in H^{0}\left(X, \omega_{X}^{-n}\right) \mid m_{p_{i}} \geq n_{i}\right\}$ where $m_{p_{i}}$ denotes the multiplicity at the point $p_{i}$ of the curve given by the divisor of zeros of the section s. The following hold:

i) $\operatorname{dim}(\mathscr{L}) \geq \frac{d\left(n^{2}+n\right)}{2}+1-\sum_{i} \frac{n_{i}^{2}+n_{i}}{2}$.

ii) If $L \in \mathscr{L}$ then $g(L) \leq \frac{d\left(n^{2}-n\right)}{2}+1-\sum \frac{n_{i}^{2}-n_{i}}{2}$.

iii) If $\pi: \mathscr{E} \rightarrow B$ is an elliptic surface obtained by blowing up $p_{1}, \ldots, p_{j}$ and $L^{\prime}$ is the strict transform of $L$ in $\mathscr{E}$ then $\operatorname{deg}\left(\left.\pi\right|_{L^{\prime}}: L^{\prime} \rightarrow B\right)=n d-\sum m_{p_{i}}$.

Proof. See [Kollár 1996, Chapter III Lemma 3.2.2].

We can now proceed to the construction of linear pencils on the $k$-minimal models. We recall that at least one point in the blow up locus of $f: \mathscr{E} \rightarrow X$ is $k$-rational, the one that comes from the contraction of the zero section. We suppose that $\mathscr{E}$ has no reducible fibers. It follows that all the points on the locus of $f$ are distinct since the blow up of infinitely near points gives rise to $(-2)$-curves, and these are always components of reducible fibers. For (i) of Theorem 1.4 it is clearly sufficient to do the construction in the case where there are two orbits by the action of the Galois group: the one of the $k$-rational point and another one with the other $d-1$ points. As in the case where $\mathbb{P}^{2}$ was a $k$-minimal model, we will look for curves satisfying the hypothesis of Lemma 5.1. 
b) A minimal model isomorphic to a del Pezzo surface of degree eight: Let $p_{2}, \ldots, p_{9}$ be the points on the blow up locus of $f: \mathscr{E} \rightarrow X$. Let $p_{2}$ be the $k$-rational point. We consider the following pencils of curves in $X$ :

$$
\begin{aligned}
& \mathscr{L}_{1}=\left\{s \in H^{0}\left(X, \omega_{X}^{-8}\right) \mid m_{p_{2}}(s) \geq 13, m_{p_{i}}(s) \geq 7 \text { for } i=3, \ldots, 9\right\}, \\
& \mathscr{L}_{2}=\left\{s \in H^{0}\left(X, \omega^{-22}\right) \mid m_{p_{2}}(s) \geq 13, m_{p_{i}}(s) \geq 23 \text { for } i=3, \ldots, 9\right\} .
\end{aligned}
$$

If $C_{1}^{\prime} \in \mathscr{L}_{1}^{\prime}$ then by Lemma 5.2

$$
g\left(C_{1}^{\prime}\right) \leq 8 \frac{(64-8)}{2}+1-\frac{(169-13)}{2}-7 \frac{(49-7)}{2}=0
$$

and $\operatorname{deg}\left(\varphi_{C_{1}}\right)=64-13-49=2$. If $C_{2}^{\prime} \in \mathscr{L}_{2}^{\prime}$ then

$$
g\left(C_{2}^{\prime}\right) \leq 8 \frac{\left(22^{2}-22\right)}{2}+1-\frac{(169-13)}{2}-7 \frac{\left(23^{2}-23\right)}{2}=0
$$

and $\operatorname{deg}\left(\varphi_{C_{2}}\right)=22.8-13-7.23=2$.

Thus $C_{1}^{\prime}$ and $C_{2}^{\prime}$ satisfy the hypothesis of Lemma 5.1. Since, by Lemma 5.2 (i), they belong to a linear pencil of curves, by Corollary 4.3 they can be chosen in a way such that the new sections induced by them in the base-changed surface are independent of the old sections and of each other.

Since there are no minimal rational surfaces of degree seven we now pass to surfaces of degree six.

c) A minimal model isomorphic to a del Pezzo surface of degree six. Let $p_{4}, \ldots, p_{9}$ be the points on the blow up locus of $f$. We consider the possible orbits under the action of the absolute Galois group. We denote the cases by $\left(n_{1}, \ldots, n_{r}\right)$ where $r$ is the number of distinct orbits and $n_{i}$ is the multiplicity of the points in the same orbit.

i) If the points lie in a $(1,2,3)$-configuration, then the blow up of the two points in the same orbit produces a surface of degree four to which we apply the constructions in e).

ii) If $\left(1,1, n_{3}, n_{4}\right)$, let $p_{4}$ and $p_{5}$ be the $k$-rational points. The blow up of $p_{4}$ produces a surface of degree five to which we can apply the constructions in d).

d) A minimal model isomorphic to a del Pezzo surface of degree five: Here we consider the pencils

$$
\begin{aligned}
& \mathscr{L}_{1}=\left\{s \in H^{0}\left(X, \omega_{X}^{-2}\right) \mid m_{p_{5}}(s) \geq 4, m_{p_{i}}(s) \geq 1 \text { for } i=6, \ldots, 9\right\} \\
& \mathscr{L}_{2}=\left\{s \in H^{0}\left(X, \omega_{X}^{-10}\right) \mid m_{p_{5}}(s) \geq 4, m_{p_{i}}(s) \geq 11 \text { for } i=6, \ldots, 9\right\} .
\end{aligned}
$$

We have

$$
\operatorname{dim}\left(\mathscr{L}_{1}\right) \geq 16-10-4=2, \quad g\left(C_{1}\right)=6-6=0,
$$

$\operatorname{deg}\left(f: C_{1}^{\prime} \rightarrow D\right)=10-4-4=2, \quad \operatorname{dim}\left(\mathscr{L}_{2}\right) \geq 5(110) / 2+1-10-4(66)=2$. 
e) A minimal model isomorphic to a del Pezzo surface of degree four: The pencils that we consider to prove Theorem 1.3 are

$$
\begin{aligned}
& \mathscr{L}_{1}=\left\{s \in H^{0}\left(X, \omega_{X}^{-1}\right) \mid m_{p_{1}}(s) \geq 2\right\}, \\
& \mathscr{L}_{2}=\left\{s \in H^{0}\left(X, \omega_{X}^{-7}\right) \mid m_{p_{1}}(s) \geq 2, m_{p_{i}}(s) \geq 8, i=2,3,4\right\} .
\end{aligned}
$$

5B. Irreducibility of the curves. We prove that if $\mathscr{E}$ satisfies the hypothesis of Theorem 1.4 the curves constructed in the previous subsection are irreducible. First, we show that a series of Cremona transformations, that is, the blow up of three distinct and noncollinear points followed by the contraction of the three lines through them, reduces each pencil of curves produced above to a pencil of lines in $\mathbb{P}^{2}$ passing through a point. Cremona transformations are not in general automorphisms of the surface, but they are automorphisms of the Picard group. In particular a class is represented by a connected curve if and only if the transformed one under a Cremona transformation is represented by an connected curve. We then use the fact that $\mathscr{E}$ has no reducible fibers to show that the curves constructed are irreducible. (See [Testa 2009] for more on irreducibility of spaces of curves on del Pezzo surfaces.)

Lemma 5.3. Let $\mathscr{E}$ be a rational elliptic surface with no reducible fibers, $X$ a $k$-minimal model of $\mathscr{E}$ as in Theorem 1.4 and let $\mathscr{L}$ be one of the pencils of curves constructed in the previous subsection. Then the generic member of $\mathscr{L}$ is an irreducible curve with geometric genus zero.

Proof. Let $d$ be the degree of $X$. Let $f: \mathscr{E} \rightarrow X$ be the map corresponding to the blow up of a $\operatorname{Gal}(\bar{k} \mid k)$-invariant set of distinct points

$$
P_{1}, \ldots, P_{d} \in X(\bar{k}) \text {. }
$$

Since $\mathscr{E}$ has no reducible fibers, the exceptional curves above $P_{i}$, for $i=1, \ldots, d$, are all independent in the Mordell-Weil group of $\mathscr{E}$. Therefore, they provide a subset of a set of generators of the Picard group of $\mathscr{E}$. We can fix a basis for the geometric Picard group of $\mathscr{E}$ to be $\left\{L_{0}, \ldots, L_{9}\right\}$ where $L_{0}$ is the total transform of a line $l$ in $\mathbb{P}^{2}, L_{1}, \ldots, L_{d}$ are the exceptional curves above $P_{1}, \ldots, P_{d}$ and $L_{d+1}, \ldots, L_{9}$ are also exceptional curves in $\mathscr{E} \times{ }_{k} \bar{k}$.

Let $g: \mathscr{E} \rightarrow \mathbb{P}^{2}$ be a blow up presentation, defined over $\bar{k}$, factoring through $f$. We represent a curve $C$ in $\mathscr{E}$ by its numerical type, that is, by the list of coordinates of its divisor class in the basis given by $\left\{L_{0}, \ldots, L_{9}\right\}$ of the Picard group:

$$
\left(d, m_{1}, \ldots, m_{9}\right),
$$

where $d$ is the degree of the image of $C$ in $\mathbb{P}^{2}$ with respect to $g: \mathscr{E} \rightarrow \mathbb{P}^{2}$, $m_{i}=m_{P_{i}}(C)$, the multiplicity of the curve $C$ at the point $P_{i}, i=1, \ldots, 9$. 
Let $C$ be a curve in $X$. Then the strict transform of $C$ through $f$ is a curve in $\mathscr{E}$ given by

$$
f^{-1}(C)-\sum_{i=1, \ldots, d} m_{p_{i}}(C) L_{i}
$$

We define the numerical type of $C$ as the numerical type of its strict transform in $\mathscr{E}$.

For example if $X=\mathbb{P}^{2}$, lines in $\mathbb{P}^{2}$ through the point $p_{1}$ are represented by the class $(1,1,0, \ldots, 0)$. If $X$ is a del Pezzo surface of degree five, curves in $\mathscr{L}_{1}^{\prime}$, where

$$
\mathscr{L}_{1}=\left\{s \in H^{0}\left(X, \omega_{X}^{-2}\right) \mid m_{P_{5}}(s) \geq 4, m_{P_{i}}(s) \geq 1, i=6, \ldots, 9\right\},
$$

are represented by $(6,2,2,2,2,4,1,1,1,1)$.

Since Cremona transformations are given by composing birational maps, to show that a curve is connected, it is sufficient to verify that curves constructed in the previous subsection can be (Cremona-)transformed into a curve whose numerical type is one of $(1,1,0, \ldots, 0), \ldots,(1,0, \ldots, 0,1)$, that is, into a line through one of the $p_{i}$.

After applying Cremona transformations successively, one can check that all curves constructed in the previous subsection are in the same class as a line through a point and are thus connected curves.

We give below the result of Cremona transformations applied to curves in $\mathscr{L}_{1}^{\prime}$ where $\mathscr{L}_{1}=\left\{s \in H^{0}\left(X, \omega_{X}^{-2}\right) \mid m_{p_{5}}(s) \geq 4, m_{p_{i}}(s) \geq 1, i=6, \ldots, 9\right\}$ and $X$ is a del Pezzo surface of degree five.

Curves in this family are encoded by $(6,2,2,2,2,4,1,1,1,1)$. Applying Cremona transformations successively, these become $(4,0,0,2,2,2,1,1,1,1)$, then $(2,0,0,0,0,0,1,1,1,1)$, and finally, $(1,0,0,0,0,0,0,0,0,1)$.

Now that we have verified that the curves constructed in the previous subsection are connected, we still have to show that the only connected component is irreducible.

Let $C$ be a curve in one of the families constructed previously. Then $C . F=2$ and $C$ has geometric genus zero. Thus if $C$ is a reducible curve then it satisfies one of the following:

i) $C=C_{1} \cup C_{2} \cup\left(F_{1}+\cdots+F_{m}\right)$ where $C_{1}$ and $C_{2}$ are sections and $F_{i}$ are components of reducible fibers.

ii) $C=D \cup\left(G_{1}+\cdots+G_{m}\right)$ where $D$ is an irreducible genus zero curve such that $D . F=2$ and $G_{j}$ are components of reducible fibers.

By Proposition 4.2, case i) can only occur for finitely many curves in a numerical family. Thus, we may suppose that the generic members of the families constructed satisfy case ii). As there are only finitely many reducible components of the fibers, the curve $D$ in case ii) is such that $\operatorname{dim}|D| \geq 1$. Since $\mathscr{E}$ has no reducible fibers, both pencils $\mathscr{L}_{1}$ and $\mathscr{L}_{2}$ constructed have irreducible generic members. 
5C. Infinitely many rational points on the new base. In order to prove the corollaries we must show that the base curve of the new elliptic surface (the base-changed one) has infinitely many $k$-rational points. To prove Corollary 1.2 one needs only one base change, thus one has to prove that infinitely many among the rational curves constructed in the previous section have a $k$-rational point. This is assured since the surface $X$ where the pencil is constructed is $k$-unirational [Manin and Tsfasman 1986, Theorem 3.5.1] so, in particular, has a Zariski dense set of $k$-rational points.

Lemma 5.4. Suppose $\mathscr{E}(k)$ is Zariski dense in $\mathscr{E}$. Let $\left\{D_{t}\right\}_{t \in \mathbb{P}^{1}}$ be a nonconstant pencil of genus zero curves defined over $k$ in $\mathscr{E}$. Then infinitely many of its curves are $k$-rational.

For Corollary 1.5 the base curve is in general an elliptic curve. Since our constructions give us families of possible new bases, we look at these families as elliptic surfaces and we show that these elliptic surfaces have a nontorsion section.

Theorem 5.5. Let $\pi: \mathscr{E} \rightarrow B$ be a rational elliptic surface defined over a number field $k$. Let $\mathscr{L}_{1}=\left\{C_{t} \mid t \in \mathbb{P}^{1}\right\}$ and $\mathscr{L}_{2}=\left\{D_{u} \mid u \in \mathbb{P}^{1}\right\}$ be two base point free linear systems of $k$-rational curves in $\mathscr{E}$ defined over $k$ with $C_{t}(k), D_{u}(k) \neq \varnothing$ for infinitely many $t, u$, such that the morphism given by the restriction of the elliptic fibration to it has degree two. Then for infinitely many $t \in \mathbb{P}^{1}(k)$ and infinitely many $u \in \mathbb{P}^{1}(k)$ we have $\#\left(C_{t} \times{ }_{B} D_{u}\right)(k)=\infty$.

Proof. Let $C \in \mathscr{L}_{1}$ be such that the base-changed elliptic surface $\mathscr{E}_{C}=\mathscr{E} \times{ }_{B} C$ has generic rank strictly larger than the generic rank of $\mathscr{E}$. The surface $\mathscr{E}_{C}$ admits a second elliptic fibration (since $\mathscr{L}_{2}$ is base point free it is not necessary to blow up points to have the fibration), namely, $\mathscr{E}_{C} \rightarrow \mathbb{P}^{1}(u)$ where $\mathbb{P}^{1}(u)$ is the index-set of the pencil of curves $\mathscr{L}_{2}$. The fibers of the latter are exactly the curves $D_{u} \times_{B} C$. We will show that infinitely many among them have positive rank by showing that this fibration is covered by an elliptic fibration of positive rank.

The natural morphism $C \rightarrow \mathbb{P}^{1}(u)$ gives us the surface $\mathscr{E}_{C} \times_{\mathbb{P}^{1}(u)} C \rightarrow C$. Fix (id, id, id) $: C \rightarrow \mathscr{\mathscr { E }}_{C} \times \mathbb{P}^{1}(u) C$ as the zero section. The involution $\iota$ on $C$ with respect to the double cover $\varphi_{C}: C \rightarrow B$ gives us another section for the fibration $\mathscr{E}_{C} \times \mathbb{P}^{1}(u) C \rightarrow C$, namely, $(\iota, \mathrm{id}, \mathrm{id})$. It intersects the zero section on the points corresponding to the ramification points $a$ and $b$ of the morphism $C \rightarrow B$. The intersection $\left(Q_{i}, Q_{i}, Q_{i}\right)$ where $Q_{i}=\varphi_{C}^{-1}\left(t_{i}\right)$, is a singular point on the fiber where it is located if and only if $t_{i}$ is also a ramification point for $\varphi_{D_{t_{i}}}: D_{t_{i}} \rightarrow B$, see [Grothendieck 1960, Corollaire 3.2.7, pp.108]. We have two possibilities:

(i) The intersection point is not a ramification point for $\varphi_{D_{t_{i}}}: D_{t_{i}} \rightarrow B$.

Since torsion sections do not meet at a nonsingular point (see for example [Miranda and Persson 1989, Lemma 1.1]) the section given by ( $\iota$, id, id) has infinite order. 
The fibration $\mathscr{E}_{C} \times_{\mathbb{P}^{1}(u)} C \rightarrow C$ has positive generic rank and thus, by NéronSilverman's specialization theorem, infinitely many fibers with positive rank. Hence the fibration $\mathscr{E}_{C} \rightarrow \mathbb{P}^{1}(u)$ has also infinitely many fibers with positive rank.

(ii) The intersection point is a ramification point for $\varphi_{D_{t_{i}}}: D_{t_{i}} \rightarrow B$. In this case the curve $C \times{ }_{B} D_{t_{i}}$ is rational. We must consider two cases:

(iia) The curve $D_{t_{i}}$ induces an independent new section in $\mathscr{E} \times{ }_{B} D_{t_{i}}$ and we are done. (iib) The generic rank of $\mathscr{E} \times{ }_{B} D_{t_{i}}$ is equal to the generic rank of $\mathscr{E}$.

Choose another curve $C \in \mathscr{L}_{1}$ in the beginning of the proof. Since only finitely many $D_{u}$ do not contribute with an extra section after base change by Corollary 4.3, if (iib) holds for almost all curves $C_{t} \in \mathscr{L}_{1}$ then the set

$$
R=\bigcup_{t \in \mathbb{P}^{1}}\left\{b \in B(k) \mid \phi_{t}: C_{t} \rightarrow B \text { is ramified above } b\right\}
$$

is finite. By Lemma 5.6 below we conclude $\mathrm{n}$ that all morphisms $\phi_{t}: C_{t} \rightarrow B$ ramify above the same points. In this case we start over by fixing a curve $D \in \mathscr{L}_{2}$ such that the surface $\mathscr{E}_{D}$ has generic rank strictly larger than the generic rank of $\mathscr{E}$. The curves $C_{t} \times{ }_{B} D$ where $C_{t}$ varies in $\mathscr{L}_{1}$ induce an elliptic fibration on $\mathscr{E}_{D}$. Since infinitely many of them contribute with a new independent section, we will be either in case (i) or case (iia).

Lemma 5.6. Let $\pi: X \rightarrow B$ be a fibration on curves from a smooth proper surface $X$ defined over a number field $k$ to a smooth proper curve $B$. Let $f: X \rightarrow \mathbb{P}^{1}$ be a genus zero fibration on $X$ such that the fibers of $f$ are not fibers of $\pi$. Let $\pi_{t}$ be the restriction of $\pi$ to the fiber $f^{-1}(t)$, for $t \in \mathbb{P}^{1}(k)$. Let

$$
R=\bigcup_{t \in \mathbb{P}^{1}}\left\{b \in B(k) \mid \pi_{t} \text { is ramified above } b\right\} .
$$

Then $R$ is either infinite or equal to $\left\{b \in B(k) \mid \pi_{t_{0}}\right.$ is ramified above $\left.b\right\}$ for any $t_{0} \in B(k)$.

5D. Proof of Theorems 1.3 and 1.4. Let $\mathscr{E}$ be as in the hypothesis of Theorem 1.3 or Theorem 1.4. Let $\mathscr{L}_{1}^{\prime}=\left\{C_{t}\right\}_{\left\{t \in \mathbb{P}^{1}\right\}}$ and $\mathscr{L}_{2}^{\prime}=\left\{D_{u}\right\}_{\left\{u \in \mathbb{P}^{1}\right\}}$ be the two pencils of rational curves constructed in the previous subsections according to the possible minimal models of $\mathscr{\mathscr { E }}$. By Corollary 4.3 all but finitely many curves $C_{t} \in \mathscr{L}_{1}$ induce a new section in $\mathscr{E}_{C}$ independent of the old sections. For each $t \in \mathbb{P}_{k}^{1}$ the pencil $\mathscr{L}_{2, t}^{\prime}=\left\{D_{u} \times{ }_{B} C_{t}\right\}$ of curves in $\mathscr{E}_{C}$ also satisfies Corollary 4.3 and thus for all but finitely many $u \in \mathbb{P}_{k}^{1}$ the curve $D_{u} \times{ }_{B} C_{t}$ induces a new section in $\mathscr{E}_{D_{u} \times{ }_{B} C_{t}}$ independent of the old sections coming from $\mathscr{E}_{C}$. Thus, after excluding finitely many $t \in \mathbb{P}^{1}$ and finitely many $u \in \mathbb{P}^{1}$, the surface $\mathscr{E}_{D_{u} \times{ }_{B} C_{t}}$ satisfies

$$
\operatorname{rk}\left(\mathscr{E}_{D_{u} \times{ }_{B} C_{t}}\left(k\left(D_{u} \times_{B} C_{t}\right)\right)\right) \geq \operatorname{rk}\left(\mathscr{E}_{C}\left(k\left(C_{t}\right)\right)\right)+1 \geq \operatorname{rk}(\mathscr{E}(k(B)))+2 .
$$


Moreover, since we excluded only finitely many of each $t$ and $u$, by Theorem 5.5 we may choose the curve $D_{u} \times{ }_{B} C_{t}$ such that it has infinitely many $k$-rational points.

Remark 14. The proof of Theorem 1.4 breaks down if the $k$-minimal model considered, $X$, is a surface of degree 6 such that the blow up locus of $f$ contains a Galois orbit with 5 points, or if it has degree 3 or 2 . Although we are still able to construct a (family of) rational curve(s) defined over the ground field, the generic member of such a family has two connected components, which cannot be used to finish the proof of the theorem. Moreover, $X$ has no families of irreducible conics (that is, rational curves intersecting the anticanonical divisor of the rational elliptic surface with multiplicity two) defined over the ground field.

\section{Corollaries from analytic number theory}

To an elliptic curve $E$ over a number field $k$, we can associate a sign $W(E \mid k)$ intrinsically via the product of local signs $W_{v}(E \mid k)$ (for a complete definition of the local sign, see for example [Rohrlich 1993]).

The parity conjecture may be stated in the following form:

Conjecture 6.1. Let $E$ be an elliptic curve over a number field $k$. Let $r$ be the rank of its Mordell Weil group. Then $W(E \mid k)=(-1)^{r}$.

Remark 15. The previous conjecture is a weak version of the Birch-SwinnertonDyer conjecture.

Over the field $\mathbb{Q}$ we know from the work of Wiles that $E$ is modular and that $W(E \mid k)$ is the sign of the functional equation of the $L$-function $L(E, s)$.

Let $\pi: \mathscr{E} \rightarrow B$ be an elliptic surface over $k$ and $U \subseteq B$ an affine open subset over which $\mathscr{E}$ is an abelian scheme. Note

$$
U_{ \pm}(k)=\left\{t \in U(k) \mid W\left(\mathscr{E}_{t}\right)= \pm 1\right\} .
$$

Modulo the parity conjecture, we also have $U_{+}(k)=\left\{t \in U(k) \mid \operatorname{rank} \mathscr{E}_{t}(k)\right.$ is even $\}$ and $U_{-}(k)=\left\{t \in U(k) \mid \operatorname{rank} \mathscr{E}_{t}(k)\right.$ is odd $\}$.

There are examples for which $W\left(E_{t}\right)$ is constant, but they all correspond to isotrivial surfaces; see for example [Cassels and Schinzel 1982]. In the nonisotrivial case and for $B \simeq \mathbb{P}^{1}, \mathrm{H}$. Helfgott [2003] has shown, under classical conjectures, that the sets $U_{ \pm}(k)$ are infinite. This is established unconditionally in some interesting cases by Helfgott [2003; 2004] and Manduchi [1995]. Much less has been done in the case $B$ is a genus one curve such that $B(k)$ is infinite. But, from previous work cited above, it seems reasonable to conjecture the following:

Conjecture 6.2. Let $\mathscr{E} \rightarrow B$ be a nonisotrivial elliptic surface defined over a number field $k$ such that $g(B)=1$ and $B(k)$ is infinite. Then $U_{+}(k)$ and $U_{-}(k)$ are infinite.

This allows us to state the following better but conditional result. 
Theorem 6.3 (modulo Conjectures 6.2 and 6.1). Let $\mathscr{E} \rightarrow B$ be a rational elliptic surface satisfying the hypothesis of Theorem 1.3. Then

$$
\#\left\{t \in B(k) \mid r_{t} \geq r+3\right\}=\infty,
$$

where $r$ is the generic rank and $r_{t}$ the rank of the fiber $\pi^{-1}(t)$.

\section{Appendix}

In this appendix we deal with rational elliptic surfaces with one nonreduced fiber.

If the blown up points are in nongeneral position, then the constructions given in Section 5A may yield reducible curves. Nevertheless, we are still able to deal with some of these cases since some of the special Galois invariant configurations of the base points of a pencil of cubic curves in the plane yield elliptic surfaces with fiber types that are easier to treat, namely, nonreduced fibers.

If the surface has a unique nonreduced fiber, then, depending on the structure of a pencil inducing $\mathscr{E}$, we will be able to prove the rank jumps for infinitely many fibers by first base-changing by a curve in $\mathscr{L}_{1}^{\prime}$ where $\mathscr{L}_{1}$ is the pencil of lines through $p_{1}$ constructed in i) of Section $5 \mathrm{~A}$. The proposition below tells us that the resulting base-changed elliptic surface is still rational and satisfies the hypothesis of Theorem 1.1, that is, it is a $k$-unirational elliptic surface.

Proposition 6.4. Let $\mathscr{E} \rightarrow B$ be a rational elliptic surface defined over a number field $k$ such that there is a $k$-birational morphism $\mathscr{E} \rightarrow \mathbb{P}^{2}$ contracting the zero section to a point $p_{1} \in \mathbb{P}^{2}(k)$. Suppose $\mathscr{E}$ has a unique fiber of type $*$, induced by a cubic curve of the form $3 m$ or $m \cup 2 l$ where $m$ is a line through $p_{1}$ and $l$ is another line.

Then for all but finitely many $L_{1} \in \mathscr{L}_{1}$ the morphism $L_{1}^{\prime} \rightarrow B$ is ramified over the place corresponding to the fiber of type $*$. Moreover, the surface $\mathscr{E} \times{ }_{B} L_{1}^{\prime}$ is $k$-unirational for all but finitely many $L_{1}^{\prime} \in \mathscr{L}_{1}^{\prime}$.

Proof. Let $F$ be the nonreduced fiber of $\mathscr{E}$ given in the hypothesis.

Suppose first that $F$ is induced by the triple line $3 m$ where $m$ is a line through $p_{1}$. Note that $p_{1}$ is a base point with multiplicity at least 3 and thus $f$ factors through the blow up of $p_{1}$ and two infinitely near points to it. The first blow up of $p_{1}$ transforms $3 m$ into $3 m^{\prime}+2 E_{1}$ where $E_{1}$ is the exceptional curve above $p_{1}$ and $m^{\prime}$ is the strict transform of $m$. The strict transform of $L_{1}$ intersects $2 E_{1}$, but does not intersect the curve $3 \mathrm{~m}^{\prime}$. The second blow up is that of $p_{1}^{\prime}$, the intersection point of $3 m^{\prime}$ and $2 E_{1}$. Since the strict transform of $L_{1}$ by the first blow up does not pass through $p_{1}^{\prime}$, this curve or its intersection with other divisors is unaffected by the remaining blow ups. Thus $L_{1}^{\prime}$, the strict transform of $L_{1}$ by $f$, intersects the multiplicity-two component of $F$ in a single point. This assures that the map $\varphi_{L_{1}}: L_{1}^{\prime} \rightarrow B$ is ramified above the place corresponding to $F$. 
Now suppose that $F$ is induced by $m \cup 2 l$ where 1 is another line. We may suppose that $L_{1}$ does not pass through other base points and hence $L_{1}$ intersects $2 l$ at a point that is not in the blow up locus of $f$. This implies that $L_{1}^{\prime}$ intersects $F$ at the double component corresponding to the strict transform of $2 l$, and thus $\varphi_{L_{1}}$ ramifies above the place corresponding to $F$.

By Lemma 2.1, $\mathscr{E} \times{ }_{B} L_{1}^{\prime}$ is rational. The existence of a nonreduced fiber in $\mathscr{E}$ assures that $\operatorname{rank}(\operatorname{Pic}(\mathscr{E}) \mid k) \geq 4$, since the components of the fiber that do not intersect the zero section contribute with at least two divisors to the Picard group over $k$. By Corollary 4.3, the rank of the surface $\mathscr{E} \times{ }_{B} L_{1}^{\prime}$ over $k$ is strictly larger than that of $\mathscr{E}$, for all but finitely many $L_{1} \in \mathscr{L}_{1}$, and thus $\operatorname{rank}\left(\operatorname{Pic}\left(\mathscr{E} \times{ }_{B} L_{1}^{\prime}\right) \mid k\right) \geq 5$. By Lemma 3.5, $\mathscr{E} \times{ }_{B} L_{1}^{\prime}$ is $k$-unirational.

We apply Theorem 1.1 to the surfaces satisfying the hypothesis of the previous proposition. This gives us the following theorem.

Theorem 6.5. Let $\mathscr{E} \rightarrow B$ be a rational elliptic surface as in Proposition 6.4. Then there is a finite covering $C \rightarrow B$ such that $C \simeq_{k} \mathbb{P}^{1}$, and the surface $\mathscr{E}_{C}=\mathscr{E} \times{ }_{B} C$ satisfies $\operatorname{rank} \mathscr{E}_{C}(k(C)) \geq \operatorname{rank}^{\mathscr{E}}(k(B))+2$.

As before, we get the following corollary.

Corollary 6.6. Let $\pi: \mathscr{E} \rightarrow B$ be an elliptic surface as in Proposition 6.4. For $t \in B(k)$, let $r_{t}$ be the rank of the fiber above the point $t$ and $r$ the generic rank. Then

$$
\#\left\{t \in B(k) \mid r_{t} \geq r+2\right\}=\infty
$$

\section{Acknowledgements}

The author would like to thank Marc Hindry for suggesting this beautiful problem and for several fruitful discussions on the topic, Damiano Testa, Daniel Bertrand, Douglas Ulmer, Jean-Louis Colliot-Thélène and Ronald van Luijk for discussions and suggestions that improved the quality of the paper. Finally the author would like to thank the referee for the careful reading of the article as well as for important suggestions that improved the readability and the quality of the paper.

\section{References}

[Billard 1998] H. Billard, "Sur la répartition des points rationnels de surfaces elliptiques", J. Reine Angew. Math. 505 (1998), 45-71. MR 99m:14039 Zbl 0948.14018

[Cassels and Schinzel 1982] J. W. S. Cassels and A. Schinzel, "Selmer's conjecture and families of elliptic curves”, Bull. London Math. Soc. 14:4 (1982), 345-348. MR 84d:14028 Zbl 0474.14010

[Grothendieck 1960] A. Grothendieck, "Éléments de géométrie algébrique, I: Le langage des schémas”, Inst. Hautes Études Sci. Publ. Math. 4 (1960), 5-228. MR 36 \#177a Zbl 0118.36206

[Helfgott 2003] H. A. Helfgott, Root numbers and the parity problem, thesis, Princeton University, 2003, Available at http://tel.archives-ouvertes.fr/docs/00/04/84/09/PDF/tel-00010129.pdf. 
[Helfgott 2004] H. A. Helfgott, "On the behaviour of root numbers in families of elliptic curves", preprint, 2004. arXiv math/0408141

[Hindry 1988] M. Hindry, "Autour d'une conjecture de Serge Lang”, Invent. Math. 94:3 (1988), 575-603. MR 89k:11046 Zbl 0638.14026

[Iskovskikh 1979] V. A. Iskovskikh, "Minimal models of rational surfaces over arbitrary fields", Izv. Akad. Nauk SSSR Ser. Mat. 43:1 (1979), 19-43. In Russian; translated in Math. USSR-Izv 14:1 (1980), 17-39. MR 80m:14021 Zbl 0412.14012

[Kollár 1996] J. Kollár, Rational curves on algebraic varieties, Ergeb. Math. Grenzgeb. (3) 32, Springer, Berlin, 1996. MR 98c:14001 Zbl 0877.14012

[Manduchi 1995] E. Manduchi, "Root numbers of fibers of elliptic surfaces", Compositio Math. 99:1 (1995), 33-58. MR 96j:11076 Zbl 0878.14028

[Manin 1966] Y. I. Manin, "Rational surfaces over perfect fields", Inst. Hautes Études Sci. Publ. Math. 30 (1966), 55-113. In Russian. MR 37 \#1373 Zbl 0171.41701

[Manin 1967] Y. I. Manin, "Rational surfaces over perfect fields, II", Mat. Sb. (N.S.) 72 (1967), 161-192. In Russian; translated in Math. USSR, Sb. 1: (1967), 141-168. MR 37 \#1374

[Manin and Tsfasman 1986] Y. I. Manin and M. A. Tsfasman, "Rational varieties: algebra, geometry, arithmetic", Uspekhi Mat. Nauk 41:2(248) (1986), 43-94. In Russian; translated in Russian Math. Surveys 41:2 (1986), 51-116. MR 87k:11065 Zbl 0621.14029

[Miranda 1980] R. Miranda, "On the stability of pencils of cubic curves", Amer. J. Math. 102:6 (1980), 1177-1202. MR 82j:14009 Zbl 0468.14009

[Miranda and Persson 1989] R. Miranda and U. Persson, "Torsion groups of elliptic surfaces", Compositio Math. 72:3 (1989), 249-267. MR 91e:14035 Zbl 0717.14019

[Néron 1956] A. Néron, "Propriétés arithmétiques de certaines familles de courbes algébriques", pp. 481-488 in Proceedings of the International Congress of Mathematicians (Amsterdam, 1954), vol. 3, edited by J. Gerretsen and J. de Groot, E. P. Noordhoff, Groningen, 1956. MR 19,321b Zbl 0074.15901

[Ribet 1979] K. A. Ribet, "Kummer theory on extensions of abelian varieties by tori", Duke Math. J. 46:4 (1979), 745-761. MR 81g:14019 Zbl 0428.14018

[Rohrlich 1993] D. E. Rohrlich, "Variation of the root number in families of elliptic curves", Compositio Math. 87:2 (1993), 119-151. MR 94d:11045 Zbl 0791.11026

[Salgado 2009] C. Salgado, Rang des surfaces elliptiques: théorèmes de comparaison, thesis, Université Denis Diderot, 2009, Available at www.math.leidenuniv.nl/ salgado/thesececi9.pdf.

[Serre 1972] J.-P. Serre, "Propriétés galoisiennes des points d'ordre fini des courbes elliptiques", Invent. Math. 15:4 (1972), 259-331. MR 52 \#8126 Zbl 0235.14012

[Shioda 1990] T. Shioda, "On the Mordell-Weil lattices", Comment. Math. Univ. St. Paul. 39:2 (1990), 211-240. MR 91m:14056 Zbl 0725.14017

[Silverman 1983] J. H. Silverman, "Heights and the specialization map for families of abelian varieties", J. Reine Angew. Math. 342 (1983), 197-211. MR 84k:14033 Zbl 0505.14035

[Silverman 1994] J. H. Silverman, Advanced topics in the arithmetic of elliptic curves, Graduate Texts in Mathematics 151, Springer, New York, 1994. MR 96b:11074 Zbl 0911.14015

[Testa 2009] D. Testa, "The irreducibility of the spaces of rational curves on del Pezzo surfaces", $J$. Algebraic Geom. 18:1 (2009), 37-61. MR 2010a:14057 Zbl 1165.14024

[Ulas 2008] M. Ulas, "Rational points on certain del Pezzo surfaces of degree one", Glasg. Math. J. 50:3 (2008), 557-564. MR 2009g:11034 Zbl 1223.14041 
Communicated by Jean-Louis Colliot-Thélène

Received 2010-11-18 Revised 2011-12-19

Accepted 2012-01-24

salgado@im.ufrj.br

Mathematisch Instituut, Universiteit Leiden, Niels Bohrweg, 1, 2333 CA Leiden, The Netherlands

Current address: Instituto de Matemática, Universidade Federal do Rio de Janeiro, 21941-909 - Rio de Janeiro, RJ, Brazil 


\section{Algebra \& Number Theory}

msp.berkeley.edu/ant

\section{EDITORS}

MANAGING EDITOR

Bjorn Poonen

Massachusetts Institute of Technology

Cambridge, USA

\author{
EDITORIAL BOARD CHAIR \\ David Eisenbud \\ University of California \\ Berkeley, USA
}

\section{BOARD OF EDITORS}

Georgia Benkart

Dave Benson

Richard E. Borcherds

John H. Coates

J-L. Colliot-Thélène

Brian D. Conrad

Hélène Esnault

Hubert Flenner

Edward Frenkel

Andrew Granville

Joseph Gubeladze

Ehud Hrushovski

Craig Huneke

Mikhail Kapranov

Yujiro Kawamata

János Kollár

Yuri Manin

Barry Mazur

Philippe Michel
University of Wisconsin, Madison, USA

University of Aberdeen, Scotland

University of California, Berkeley, USA

University of Cambridge, UK

CNRS, Université Paris-Sud, France

University of Michigan, USA

Freie Universität Berlin, Germany

Ruhr-Universität, Germany

University of California, Berkeley, USA

Université de Montréal, Canada

San Francisco State University, USA

Hebrew University, Israel

University of Virginia, USA

Yale University, USA

University of Tokyo, Japan

Princeton University, USA

Northwestern University, USA

Harvard University, USA

École Polytechnique Fédérale de Lausanne
Susan Montgomery

Shigefumi Mori

Raman Parimala

Jonathan Pila

Victor Reiner

Karl Rubin

Peter Sarnak

Joseph H. Silverman

Michael Singer

Vasudevan Srinivas

J. Toby Stafford

Bernd Sturmfels

Richard Taylor

Ravi Vakil

Michel van den Bergh

Marie-France Vignéras

Kei-Ichi Watanabe

Andrei Zelevinsky

Efim Zelmanov
University of Southern California, USA

RIMS, Kyoto University, Japan

Emory University, USA

University of Oxford, UK

University of Minnesota, USA

University of California, Irvine, USA

Princeton University, USA

Brown University, USA

North Carolina State University, USA

Tata Inst. of Fund. Research, India

University of Michigan, USA

University of California, Berkeley, USA

Harvard University, USA

Stanford University, USA

Hasselt University, Belgium

Université Paris VII, France

Nihon University, Japan

Northeastern University, USA

University of California, San Diego, USA

\section{PRODUCTION}

production@msp.org

Silvio Levy, Scientific Editor

See inside back cover or www.jant.org for submission instructions.

The subscription price for 2012 is US \$175/year for the electronic version, and \$275/year ( $\$ 40$ shipping outside the US) for print and electronic. Subscriptions, requests for back issues from the last three years and changes of subscribers address should be sent to Mathematical Sciences Publishers, Department of Mathematics, University of California, Berkeley, CA 94720-3840, USA.

Algebra \& Number Theory (ISSN 1937-0652) at Mathematical Sciences Publishers, Department of Mathematics, University of California, Berkeley, CA 94720-3840 is published continuously online. Periodical rate postage paid at Berkeley, CA 94704, and additional mailing offices.

ANT peer review and production are managed by EditFLOW ${ }^{\circledR}$ from Mathematical Sciences Publishers.

PUBLISHED BY

mathematical sciences publishers

http://msp.org/

A NON-PROFIT CORPORATION

Typeset in IATEX

Copyright ( 2012 by Mathematical Sciences Publishers 


\section{Algebra \& Number Theory}

\section{Volume $6 \quad$ No. $7 \quad 2012$}

On the rank of the fibers of rational elliptic surfaces

Cecília Salgado

Néron's pairing and relative algebraic equivalence

CÉDRIC PÉPIN

Free subalgebras of quotient rings of Ore extensions

JASON P. BELL and DANIEL ROGALSKI

Classes de cycles motiviques étales

1369

BRUNO KAHN

Higher-order Maass forms

1409

ROELOF BRUGGEMAN and NiKOLAOS DIAMANTIS

Log canonical thresholds, $F$-pure thresholds, and nonstandard extensions

Bhargav Bhatt, Daniel J. Hernández, Lance Edward Miller and Mircea MUSTAȚĂ

The semistable reduction problem for the space of morphisms on $\mathbb{P}^{n}$

ALON LEVY

Grothendieck's trace map for arithmetic surfaces via residues and higher adèles

MATTHEW MORROW

Crystalline extensions and the weight part of Serre's conjecture

Toby GeE, Tong LiU and DAVID SAVITT

Annihilating the cohomology of group schemes 